\title{
Vaginal Wall Leiomyoma: A Case Report
}

\author{
RABEYAAKTHER ${ }^{\mathrm{a}}$, MD. NOWFEL ISLAM $^{\mathrm{b}}$, HASINAAFROZ $^{\mathrm{c}}$
}

\section{Summary:}

Vaginal wall leiomyoma is rare. They are typically located in the anterior or lateral wall of the vagina. Approximately 301 cases have been reported in the literature. Usually patients with vaginal leiomyoma present with a mass per vaginum or dyspareunia or pressure symptoms on the urinary tract. However, sometimes it is difficult to diagnose preoperatively because of an unusual presentation. History and a careful clinical examination may help to rule out malignancy. Here, we report a case of vaginal leiomyoma which was incidentally identified during routine gynecological check up of a 36-

\section{Introduction:}

Vaginal tumors are rare and include cysts of vestigial structures, implantation dermoid, endometriotic cyst, papilloma, hemangioma, mucus polyp, adenoma and rarely leiomyoma. Approximately 301 cases have been reported in the literature. It was first detected in 1733 by Denys de Leyden ${ }^{1}$. Bennett and Erlich ${ }^{2}$ found only nine cases in 50,000 surgical specimens and only one case in 15,000 autopsies reviewed at Johns Hopkins Hospital. These tumors arise most commonly from the anterior vaginal wall causing varied clinical presentations. They may or may not be associated with leiomyomas elsewhere in the body. We report a case of primary leiomyoma of vagina arising from lateral wall and it was diagnosed incidentally during vaginal examination.

\section{Case Report:}

A 36-year-old female, para 2, came to the outpatient department for routine gynecological check up. A mass was felt during per vaginal examination in the left lateral vagina wall (Figure - 1), but the upper limit of the mass

a. Dr. Rabeya Akther, Deputy Chief Medical Officer, Bangladesh Bank.

b. Prof. Md. Nowfel Islam, Professor, Pathology Department, National institute of Neurosciences \& Hospital, Dhaka

c. Dr. Hasina Afroz, Consultant, Obstetrics \& Gynecology Department ,United Medical College \& Hospital, Dhaka.

Address of Correspondence: Dr. Rabeya Akther, Chief Consultant, Bangladesh Bank Medical Center, Motijheel, Dhaka, Mobile: 01817517100,E-mail: rabeya.akter@bb.org.bd

Received: 1 Dec., 2018

Accepted: 19 July, 2019 year-old multiparous lady as a large, solid, painless mass in the left vaginal wall. The overlying mucosa moved freely over the mass. The USG revealed nothing abnormal in the pelvic organs. Surgical enucleation was done vaginally and subsequent histopathology showed irregular and whorling bundles of smooth muscle cells confirming vaginal leiomyoma.

Keywords: Leiomyoma, vaginal leiomyoma, benign uterine leiomyoma.

(J Bangladesh Coll Phys Surg 2019; 37: 209-211) DOI: http://dx.doi.org/10.3329/jbcps.v37i4.43353

could not be delineated. The mass was hard inconsistency, non tender and of restricted mobility. Vaginal mucosa moved freely over the mass. There was no history of dysperunia, dysuria, increased frequency, or any feature of urinary retention. No mass was palpable in the groin. An ultrasonogram was done which revealed no abnormality. Considering it as a case of enlarged lymph node, the mass was removed surgically by vaginal route. A $4.5 \mathrm{~cm} \times 3.5 \times 2.5 \mathrm{~cm}$ sized solid tumor was found. (Figure 2,3) Vaginal wall was closed in layers. To prevent bleeding or oozing a vaginal pack was given. A Foley's catheter was introduced in the urethra for protecting the latter. The tumor was then sent for histopathological examination with a presumptive diagnosis of vaginal leiomyoma. Gross examination revealed a $4.5 \times 3.5 \times 2.5 \mathrm{~cm}$

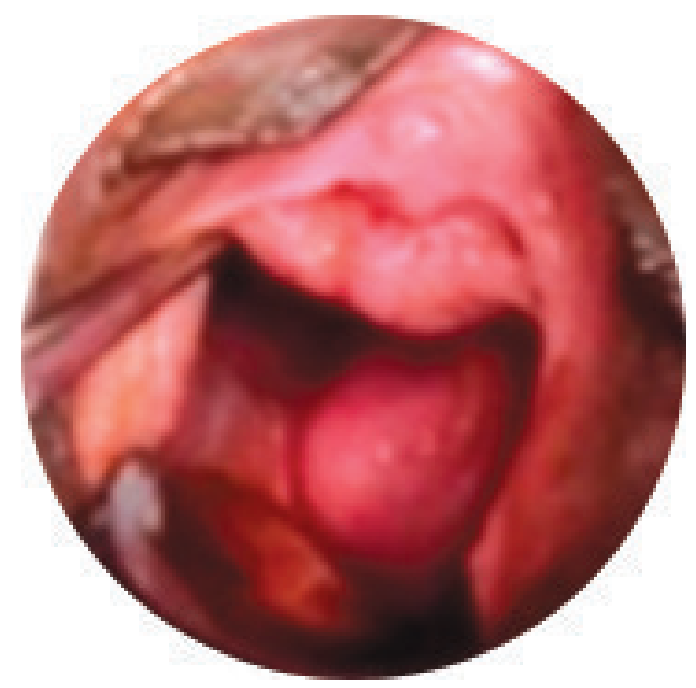

Fig.-1: photograph showing a mass in the vagina. 
solid mass with a whorling appearance in the cut section. Microscopic examination showed irregular and whorling bundles of smooth muscle cells. (Figure 4, 5, 6). No malignancy was seen. Final diagnosis was leiomyoma.

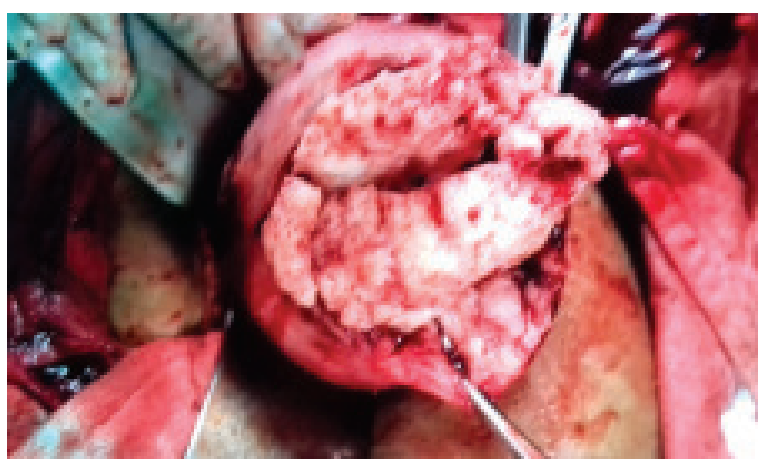

Fig.-2: Photograph showing a mass in the vagina (during operation).

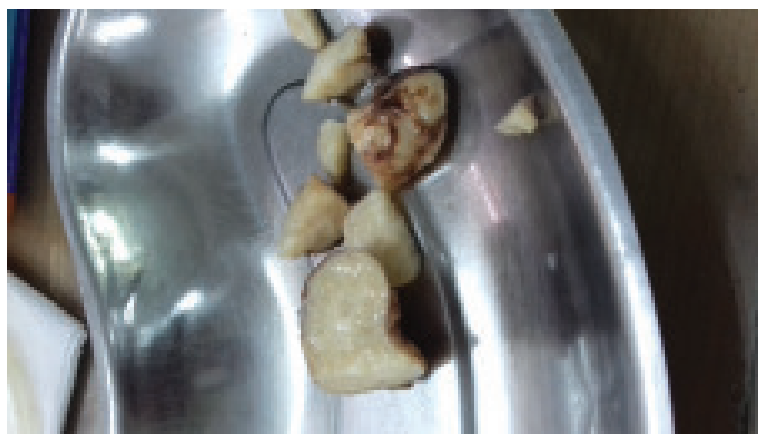

Fig.- 3: Photograph showing the whorling pattern on cut section of the vaginal leiomyoma

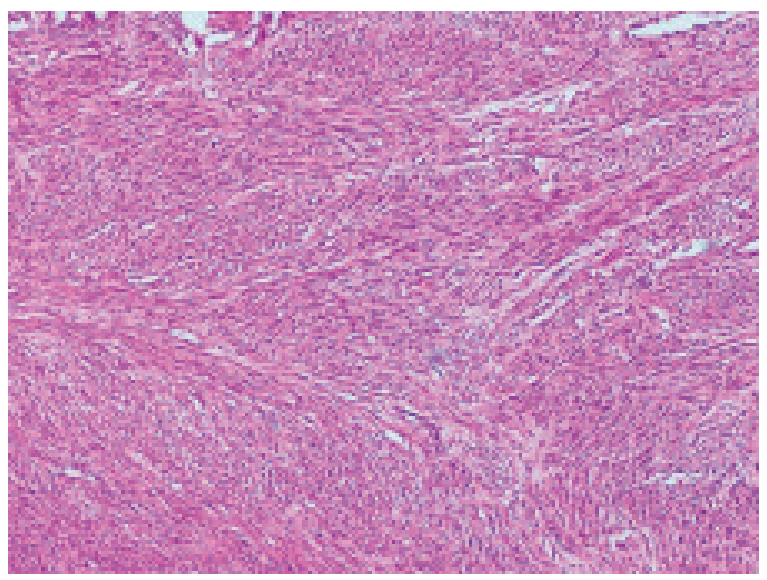

Fig.- 4: Microphotograph showing the leiomyoma (hematoxylin and eosin stain, $\times 10$ magnification)

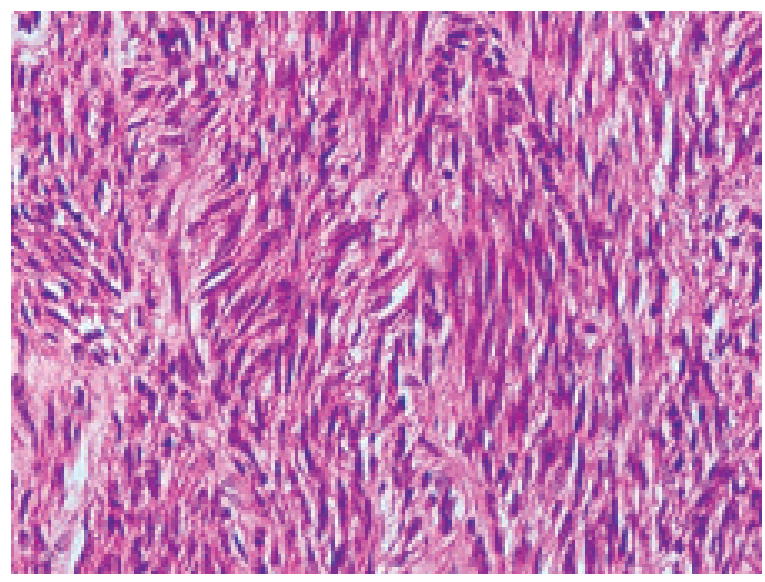

Fig.-5: Microphotograph showing the leiomyoma (hematoxylin and eosin stain, $\times 40$ magnification)

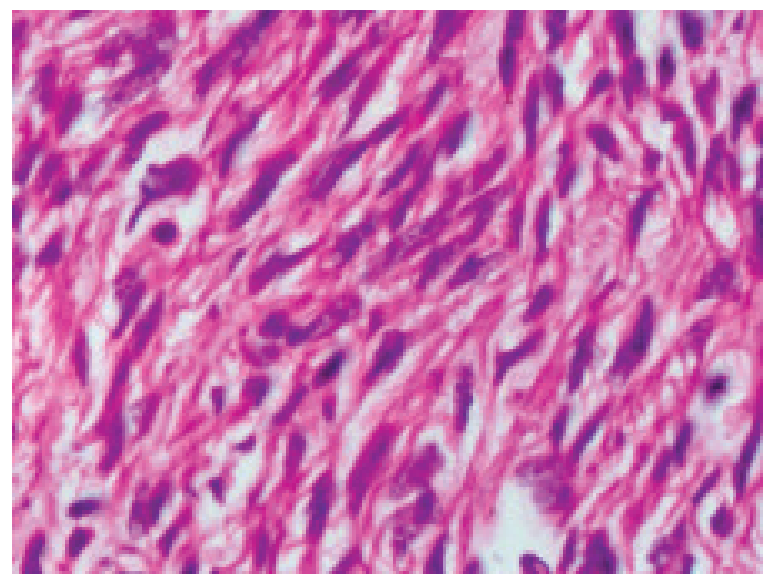

Fig.- 6: Microphotograph showing the leiomyoma (hematoxylin and eosin stain, $\times 100$ magnification)

\section{Discussion:}

Tumors of vagina are rare and there are only around 301 reported cases of vaginal leiomyomas since the first described case in 1733 by Denys de Leyden ${ }^{1}$.Leiomyomas in female genital tract are common in the uterus and to some extent in the cervix followed by the round ligament, utero-sacral ligament, ovary, and inguinal canal ${ }^{1}$. Occurrence in vagina is very rare. Vaginal leiomyomas are commonly seen in the age group ranging from 35 to 50 years and are reported to be more common among Caucasian women ${ }^{1,2}$. Usually occurs as single, well-circumscribed mass arising from the midline anterior wall and less commonly, from the posterior and lateral walls ${ }^{1}$. They may be asymptomatic but depending on the site of occurrence, they can give rise to varying symptoms including lower abdominal pain, low back 
pain, vaginal bleeding, dyspareunia, and frequency of micturition, dysuria, or other features of urinary obstruction. These tumors can be intramural or pedunculated and solid as well as cystic. Usually these tumors are single, benign, and slow growing but sarcomatous transformation has been reported ${ }^{1,3}$. Preoperatively, diagnosis by ultrasonography may be difficult, but magnetic resonance imaging usually clinches the diagnosis. In magnetic resonance imaging, they appear as well-demarcated solid masses of low signal intensity in T1 and T2 weighted images, with homogenous contrast enhancement, while leiomyosarcomas and other vaginal malignancies show characteristic high T2 signal intensity with irregular and heterogeneous areas of necrosis or hemorrhage 2,3 . However, histopathological confirmation is the gold standard of diagnosis and also beneficial to rule out any possible focus of malignancy. Surgical removal of the tumor through vaginal approach, preferably with vaginal packing and urethral catheterization to prevent vaginal bleeding in postoperative period is recommended.

\section{Reference:}

1. Indranil Chakrabarti, Anuradha De, and Shyamapada Pati: Vaginal leiomyoma. J Midlife Health. 2011 Jan-Jun; 2(1): $42-43$.

2. Elsayes KM, Narra VR, Dillman JR, Velcheti V, Hameed O, Tongdee R, et al. Vaginal Masses: Magnetic Resonance Imaging Features with Pathologic Correlation. Acta Radiol. 2007; 8:921-33. [PubMed]

3. Bae J H, Choi SK, Kim JW. Vaginal leiomyoma: A case report and review of the literature. J Women's Med. 2008; $1: 92-4$. 